\title{
Optical time domain reflectometer for precision measurement of signal delay in optical fiber
}

D. Prokhorov, S. Donchenko, O. Kolmogorov, E. Chemesova

D. Prokhorov, S. S. Donchenko, O. V. Kolmogorov, E. V. Chemesova, "Optical time domain reflectometer for precision measurement of signal delay in optical fiber," Proc. SPIE 11057, Modeling Aspects in Optical Metrology VII, 1105714 (21 June 2019); doi: 10.1117/12.2530073

SPIE. Event: SPIE Optical Metrology, 2019, Munich, Germany 


\title{
Optical time domain reflectometer for precision measurement of signal delay in optical fiber
}

\author{
D.V. Prokhorov, S.S. Donchenko*, O.V. Kolmogorov, E.V. Chemesova \\ VNIIFTRI, Mendeleyevo, Russia, 141570
}

\begin{abstract}
The overview of different types of reflectometers is given.

The theoretical calculation of factors, such as instability of internal reference clocks and resolution of time interval measurement device, influencing on resolution and uncertainty of signal delay (which can be calculated into length) is given.

One of the ways to decrease uncertainty type A of distance measurements is suggested scheme of pulse reflectometer with multistop event timer. Due to ability of measurement averaging it is possible to reduce resolution less then $0.1 \mathrm{~mm}$.

In the work principle of operation of the proposed reflectometer and functional scheme are given. The impact of such factors as chromatic and polarization mode dispersion, timer trigger error, temperature fluctuations are considered. The requirements to laser module due to dispersion characteristics of measured fiber are given. The temperature variation affects mostly on signal delay in photodetector and other electronics. It also changes the delay of optical pulse in fiber coil, which is used for dead zone riddance. The results of theoretical error limits calculations for such reflectometers are presented.

The results of experimental studies of reflectometer are presented. It is shown that the proposed scheme of the optical time domain reflectometer and technical solutions make possible the signal propagation delays measurements with an resolution better than $5 \mathrm{ps}$ and combined uncertainty less than $100 \mathrm{ps}$.
\end{abstract}

Keywords: Optical time domain reflectometry, optical fiber, time scales, signal propagation delay

\section{INTRODUCTION}

Systems of time scales comparisons and synchronization [1], using fiber optic lines, acquire high accuracy of determining the signal propagation delays in optical fibers (optical fibers, passive optical elements). Thus, the measurement uncertainty of the signal delay should not exceed $\pm 100 \mathrm{ps}$, for systems transmitting signals over several fibers, the uncertainty of determining the difference in delay between the fibers should not exceed $\pm 20 \mathrm{ps}$.

Time domain reflectometers which are used for monitoring telecommunication fiber-optic fiber lines [2] do not allow to solve the problem of controlling signal delays in fiber optic comparison and synchronization systems, because they have a maximum resolution of $0.4-0.6 \mathrm{~m}$ when measuring length, which corresponds to a resolution of 2-3 nanoseconds if signal propagation delay is measured.

Characteristics of various types of optical reflectometers are shown in Table 1. To measure the signal delay in optical fiber links with a resolution better than $20 \mathrm{~mm}$, complex and expensive instruments are needed (for example, LUNA, USA)

*donchenko_ss@vniiftri.ru; phone7 9264221648.

Modeling Aspects in Optical Metrology VII, edited by Bernd Bodermann, Karsten Frenner

Proc. of SPIE Vol. 11057, 1105705, (C) The Authors. Published Under a Creative

Commons Attribution CC-BY 3.0 License · doi: 10.1117/12.2530073 
Table 1. Characteristics of optical reflectometers

\begin{tabular}{|c|c|c|c|}
\hline Reflectometer & Measurement range, $\mathrm{km}$ & Dead zone, $\mathrm{m}$ & 600 \\
\hline $\begin{array}{c}\text { EXFO OTDR FTB- } \\
\text { 7200D }\end{array}$ & $\begin{array}{c}0 \ldots 10 \text { (range for max } \\
\text { resolution) }\end{array}$ & 1 & 125 \\
\hline FOD 7302 & $\begin{array}{c}0 \ldots 2 \text { (range for max } \\
\text { resolution) }\end{array}$ & 1.5 & $0,01-1$ \\
\hline LUNA OBR 4600 & $0,03-2$ & - & 5 \\
\hline OFM 130 & $0 \ldots 20$ & 0.2 & $\mathrm{~mm}$ \\
\hline
\end{tabular}

The lack of high-precision LUNA reflectometers is a small distance range (up to $2 \mathrm{~km}$ of an optical cable), since they are intended mainly for the control of airborne fiber optic lines and passive optical elements. Telecommunication reflectometers do not provide the accuracy required for the propagation delay measurement of a signal even in fibers up to $1 \mathrm{~km}$ long, which are required for the time scales comparison and synchronization systems. Increase of the measured fiber length, leads to increase of the uncertainty of the delay determining. When measuring length up to $100 \mathrm{~km}$ in the forward and reverse direction - relative frequency offset (reference frequency of quartz oscillator in OTDR) from $10^{-5}$ to $5 \cdot 10^{-5}$ corresponds to delay measurement uncertainty from 10 to $50 \mathrm{~ns}$. Thus, the existing commercially available optical reflectometers do not allow to measure signal delays in fiber optic lines up to $100 \mathrm{~km}$ long with an uncertainty less than $100 \mathrm{ps}$, which leads to develop a new reflectometer to solve this problem.

The frequency method of reflectometry [3,4] can be used for accurate determination of delays in fiber-optic communication lines; however, the disadvantage of this method is demand in low noise level of optical signal.

The resolution of the pulse reflectometers depends in the main on the characteristics of the time interval counters: the single-shot RMS resolution and the frequency uncertainty of the reference oscillator. The most accurate modern time interval counters have a resolution of about $10 \mathrm{ps}$. Therefore, time interval counters with picosecond resolution can be used for measurement of signal propagation delays with an uncertainty of less than $100 \mathrm{ps}$.

\section{OPERATION PRINCIPAL}

The proposed scheme of a pulsed optical reflectometer, built using a time interval counter with picosecond resolution (event timer) - EventTimer A033ET / USB is shown on figure 1.

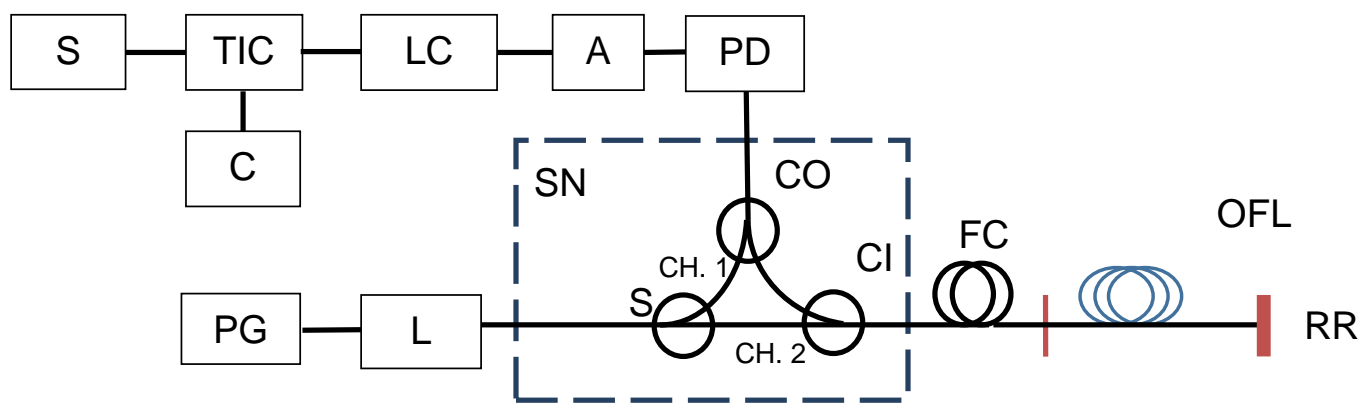

Figure 1. Scheme of optical time domain reflectometer. S - frequency standard, TIC - time interval counter, C computer, PG - pulse generator, L - laser, LC - logical converter, A - amplifier, PD - photodetector, SN - splitter node, $\mathrm{S}$ - splitter, CO - coupler, CI - circulator, FB - fiber coil, FOL - fiber optical link, RR - retroreflector. 
The operation principle of the proposed reflectometer is as follows. The PG pulse generator forms electric pulses, which trigger laser module L. Optical pulses from the laser module output enter the SN splitter node, where the pulse is divided into two channels. It enters the first photodetector PD, amplified by amplifier A, and converted using the logical converter LC to the required pulse form. This pulse enters the input of TIC time event counter, and it captures the moment of emission of the pulse $\mathrm{T}_{\mathrm{i} 1}$. The pulse generator is used by the TIC as a source of the reference frequency signal. In the second channel, the pulse passes through the built-in FB fiber coil into the measured fiber optic line and propagates through it. Reflected from the retroreflector RR, placed at the fiber end, the pulse passes through the fiber in the opposite direction and through the circulator $\mathrm{C}$ enters the photodetector, while the TIC records the moment of arrival of the reflected pulse $T_{i 2}$. The measurement accuracy can be improved by averaging the measurement results, the listed operations are repeated $\mathrm{N}$ times $(\mathrm{i}=1 \ldots \mathrm{N})$. The two-directional delay of pulse propagation $\mathrm{T}_{\mathrm{p}}$ in the test fiber in the forward and reverse direction (two-way delay) is determined by the formula [1]:

$$
T_{p}=\frac{1}{N} \sum_{i=1}^{N}\left(T_{i 2}-T_{i 1}+\tau_{c}\right),
$$

where $\tau_{\mathrm{c}}$ is the reflectometer instrumental correction, which takes into account the difference in delays in two channels of the SN and the delay in the built-in FB used to eliminate the "dead zone". By measuring the two-way propagation delay, fiber length can be calculated using the refractive index data.

\section{UNCERTAINTY BUDGET}

The measurement uncertainty estimate of the reflectometer according to the proposed scheme (Figure 1) is listed below. The sources of uncertainty are: the trigger uncertainty of the event timer counter; the uncertainty in determining the instrumental correction $\theta \tau$; the uncertainty of the signal delay in the receiving path due to the influence of temperature changes $\theta_{\mathrm{T}}$; the correction uncertainty, accounting the effect of attenuation and dispersion in the optical fiber of the pulse's front $\theta_{\mathrm{p}}$; the uncertainty of for the source wavelength correction $\theta \lambda$, the uncertainty due to the longterm (for 1 year) frequency instability of the reference generator $\theta$ s, the uncertainty due to the length change of the FB depending on the ambient temperature $\theta \kappa$.

The contribution of the generator instability of the is not more than $10^{-8}$ when measuring fiber optic to $100 \mathrm{~km}$ it is less than $10 \mathrm{ps}$, so it can be ignored.

The change in the delay because of length variance of the FB can be determined by the formula:

$$
\Delta t=L \cdot \frac{\Delta n(T)}{c}+\Delta L(T) \cdot \frac{n}{c},
$$

where $\Delta t$ is the fiber delay change; $\mathrm{L}$ is the length of the optical fiber; $\mathrm{n}$ is the refractive index; $\mathrm{c}$ is the speed of light in vacuum; $\Delta \mathrm{n}(\mathrm{T})$ - the change in the refractive index under the effect of temperature changes; $\Delta \mathrm{L}(\mathrm{T})$ is the fiber length change due to temperature changes. The calculation results are shown in Figure 2.

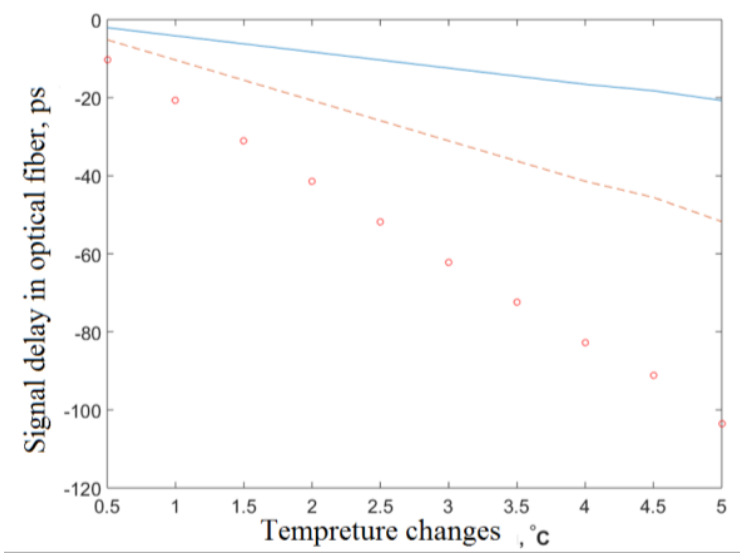

Figure 2. Changes in the propagation delay of a signal in a FOCL depending on the ambient temperature. 
The solid line shows the delay for fiber optic $1000 \mathrm{~m}$ long, dotted $-500 \mathrm{~m}$ long, dots - 200m long. As it can be seen from the figure 2 - temperature fluctuations during measurements up to $5^{\circ} \mathrm{C}$ in a $200 \mathrm{~m}$ fiber coil the delay will be less than 20 ps. Therefore, in calculations and experiments, a fiber coil with a length of less than $200 \mathrm{~m}$ will be used for reflectometer.

When measuring the signal propagation delay in the fiber optical link, one should also take into account that the obtained value will be used in the time scales systems of comparison and synchronization. The source wavelength in these systems will differ from the one, which was used during measurements, performed on the reflectometer. Considering the fiber is SMF-28, for which the dispersion value D $(\lambda)$ for the wavelength of $1550 \mathrm{~nm}$ should not exceed $18\left[\frac{p s}{n m \cdot k m}\right]$.

Then, the difference between the wavelengths of the laser sources at $1 \mathrm{~nm}$ will cause the difference in the propagation delays of the signals in optical fiber link $35 \mathrm{~km}$ long up to $630 \mathrm{ps}$, for a optical fiber link with a length of $100 \mathrm{~km}$ it is $1800 \mathrm{ps}$. As can be seen from the calculation, the difference between the wavelengths of the sources of the reflectometer and the time scales comparison and transmission systems can make a large contribution to uncertainty type B- $\theta_{\lambda}$. The value of $\theta_{\lambda}$ can be reduced in two ways.

The same optical source from can be used both in systems of time scales comparison and transmission and in the reflectometer, if its parameters meet the requirements for the radiation source. The disadvantage of this approach is the need to calibrate the delays in the OTDR, the advantage is the exception of the $\theta \lambda$.

In the case when the lasers are different in the reflectometer and time scales comparison and transmission systems of it is necessary to measure the wavelength of the sources of laser radiation and use correction if they differ. The table below shows the characteristics of commercial optical spectrum analyzers.

Table 2 - Wavelength accuracy of optical spectrum analyzers

\begin{tabular}{|c|c|}
\hline Optical spectrum analyzer & Wavelength accuracy \\
\hline Anritsu MS 9740A & $\pm 20 \mathrm{pm}$ \\
\hline EXFO FTB 5230S & $\pm 20 \mathrm{pm}$ \\
\hline Viavi OSA-500RS & $\pm 10 \mathrm{pm}$ \\
\hline
\end{tabular}

For the calculation of $\theta \lambda$, we will assume that the wavelength measurement uncertainty is $20 \mathrm{pm}$ (the uncertainty of the majority of optical spectrum analyzers). Then $\theta \lambda$ for fiber optic $100 \mathrm{~km}$ long will be $36 \mathrm{ps}$.

Components of the reflectometer's uncertainty budget are shown in the table 3 .

Table 3 - Reflectometer's uncertainty budget

\begin{tabular}{|c|c|c|c|}
\hline Source of uncertainty & $\begin{array}{c}\text { Value for distance up to } \\
3 \mathrm{~km}\end{array}$ & $\begin{array}{c}\text { Value for distance up to } \\
50 \mathrm{~km}\end{array}$ & $\begin{array}{c}\text { Value for distance up to } \\
100 \mathrm{~km}\end{array}$ \\
\hline$\theta_{t r}$ & $\pm 5 \mathrm{ps}$ & $\pm 5 \mathrm{ps}$ & $\pm 5 \mathrm{ps}$ \\
\hline$\theta_{\tau}$ & $\pm 5 \mathrm{ps}$ & $\pm 5 \mathrm{ps}$ & $\pm 2 \mathrm{ps}$ \\
\hline$\theta_{T}^{*}$ & $\pm 2 \mathrm{ps}$ & $\pm 2 \mathrm{ps}$ & $\pm 15 \mathrm{ps}$ \\
\hline$\theta_{p}$ & - & $\pm 7 \mathrm{ps}$ & $\pm 10 \mathrm{ps}$ \\
\hline$\theta_{f}$ & - & $\pm 5 \mathrm{ps}$ & $\pm 36 \mathrm{ps}$ \\
\hline$\theta_{\lambda}$ & $\pm 0,36 \mathrm{ps}$ & $\pm 18 \mathrm{ps}$ \\
\hline
\end{tabular}

$*$ at temperature instability within $\pm 1^{\circ} \mathrm{C}$ 
To test the performance of the reflectometer setup and estimation of its resolution, test lines were assembled from the $1 \mathrm{~km}$ optical fiber coil and the optical junction (a short segment of optical fiber in a ceramic shell placed in a metal case) were used. The geometric transition length, previously measured with a micrometer, was $26.2 \mathrm{~mm}$ (Figure $3)$.

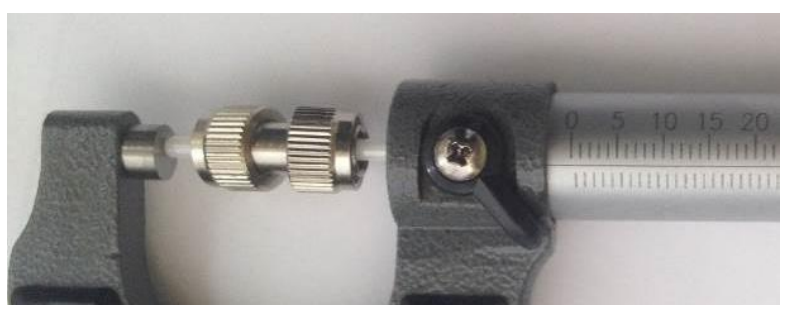

a)

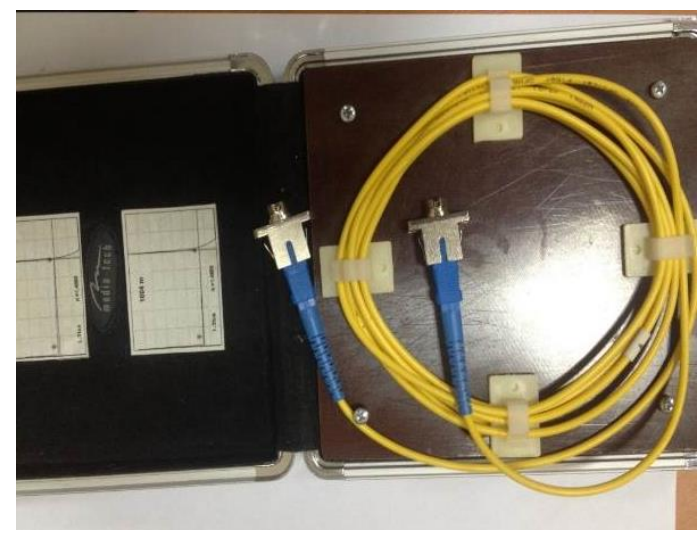

б)

Figure 3. a) Measurement of the length of the fiber transition, b) test line

Further measurements were carried out at $1310 \mathrm{~nm}$ wavelength in two stages: first, the OTDR measured the pulse propagation delay in the test line consisting only of a fiber coil, and then the optical junction was connected to the end of the test line and the total delay in the composite test line was measured. The instability of the temperature in the room did not exceed $\pm 1^{\circ} \mathrm{C}$.

For a fiber coil, the average value of the signal propagation delay is $9.8493263 \mu \mathrm{s}$, and for a fiber coil with an optical transition $-9.8495758 \mu \mathrm{s}$, the difference between the results is $0.0002495 \mu \mathrm{s}$, which is $25.5 \mathrm{~mm}$ in terms of the geometric length of the optical junction. The length of this optical junction, measured with a micrometer, was $26.2 \mathrm{~mm}$, and the difference between the values is $0.7 \mathrm{~mm}$.

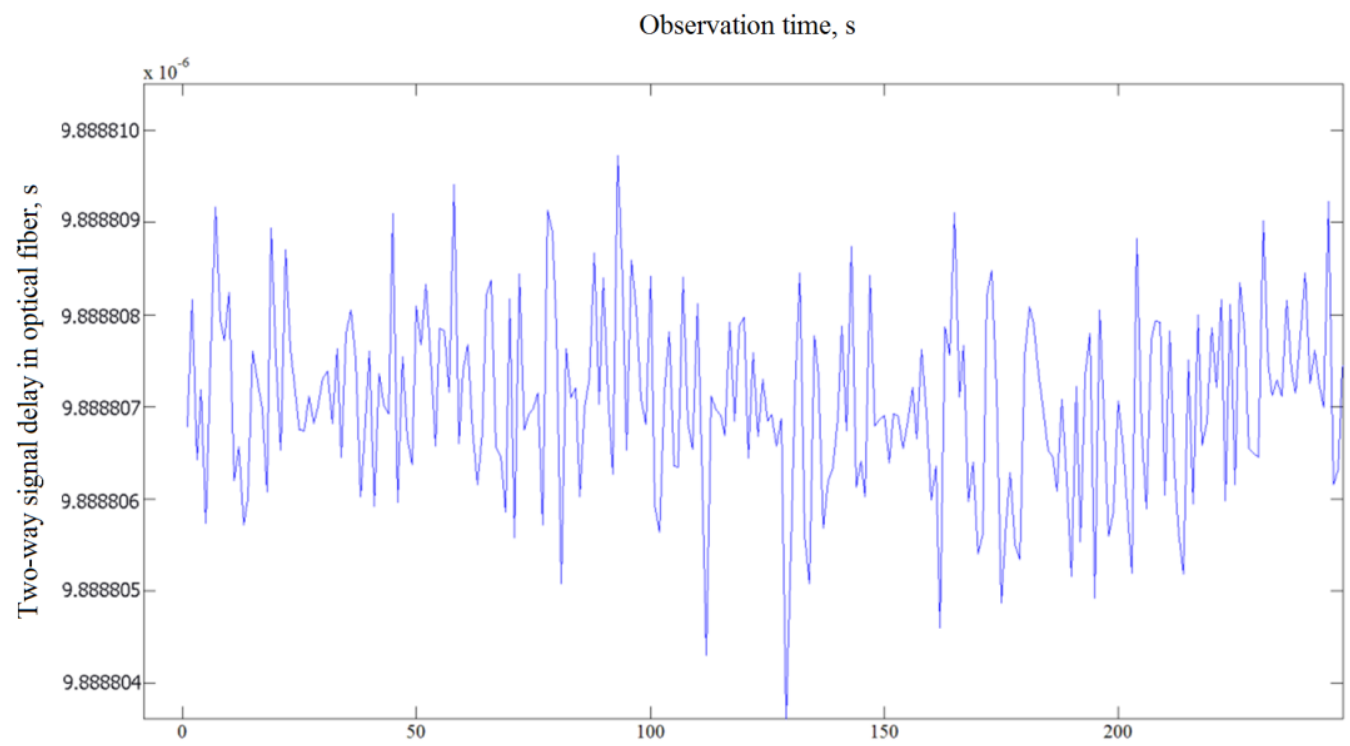

Figure 4. Results of delay measurements in optical fiber 
Standard deviation $\sigma$ of the reflectometer's setup measurement results do not exceed 1.2 ps. Based on the obtained results, the reflectometer's resolution (the minimum difference in delays that the device can register) estimation was carried out. Experimental studies of the OTDR showed that resolution doesn't not exceed 5-8 ps (1-1.5 mm in terms of length), and the uncertainty of determining the difference in signal propagation delays is no more than $16 \mathrm{ps}$.

Practical testing of the reflectometer model was carried out while measuring the delay in a real optical fiber line between separate buildings ( $1.2 \mathrm{~km}$ apart). The standard deviation of the measurement results do not exceed $5 \mathrm{ps}$.

Taking into account the above, the combined uncertainty for signal delay measurements up to $100 \mathrm{~km}$ can be calculated using formula:

$$
u_{c}=\sqrt{\sigma^{2}+\frac{\theta^{2} t r}{3}+\theta^{2} \tau+\frac{\theta^{2} T}{3}+\frac{\theta^{2} p}{3}+\frac{\theta^{2} f}{3}+\frac{\theta^{2} \lambda}{3}}=25 \mathrm{ps}
$$

\section{Conclusion}

Thus, for high-precision determination of signal propagation delays in an optical fiber, it is proposed to use the method of pulsed reflectometry in the time domain with time interval counter (picosecond resolution). A feature of this scheme is the usage of a short built-in fiber coil the reflectometer, which allows to eliminate the dead zone at the beginning of the scale and determining the current value of the instrumental correction. Theoretical and experimental studies have shown that the reflectometer, constructed according to the proposed scheme, measures the signal propagation delays in optical fibers with an error of no more than $\pm 25 \mathrm{ps}$.

\section{REFERENCES}

[1] Donchenko S.S., Kolmogorov O.V., Prokhorov D.V., "A SYSTEM OF ONE- AND TWO-WAY COMPARISONS OF TIME SCALES”, Measurement Techniques, V. 58. № 1. 18-22 (2015).

[2] Kunihiro, T. and Fumihiko I, «Recent Research and Development of Optical Fiber Monitoring in Communication Systems», Photonic Sensors Vol. 3, No. 4: 304-313 (2013).

[3] Jiong, L., Jiulin, G., Zhishen Z, Xiaobo H., Changsheng Y., QI Q., Shanhui X., Zhongmin Y., "High spatial resolution distributed fiber strain sensor based on phase-OFDR", OPTICS EXPRESS, Vol. 25, No. 22 (30): 1-10, (2017).

[4] Liokumovich, L. B, Ushakov N., Kotov O. I., Bisyarin, M. A., Arthur H. Hartog. "Fundamentals of optical fiber sensing schemes based on coherent optical time domain reflectometry: Signal model under static fiber conditions September”, Journal of Lightwave Technology 33(17):3660-3671 (2015). 